\title{
The Functional Consequences of Social Attention for Memory-guided Attention Orienting and Anticipatory Neural Dynamics
}

\author{
Brianna Ruth Doherty ${ }^{1,3}$, Freek van Ede ${ }^{1}$, Alexander Fraser ${ }^{1}$, Eva Zita Patai $^{2}$, \\ Anna Christina Nobre ${ }^{1}$, and Gaia Scerif ${ }^{1}$
}

\begin{abstract}
Social attention when viewing natural social (compared with nonsocial) images has functional consequences on contextual memory in healthy human adults. In addition to attention affecting memory performance, strong evidence suggests that memory, in turn, affects attentional orienting. Here, we ask whether the effects of social processing on memory alter subsequent memory-guided attention orienting and corresponding anticipatory dynamics of $8-12 \mathrm{~Hz}$ alpha-band oscillations as measured with EEG. Eighteen young adults searched for targets in scenes that contained either social or nonsocial distracters and their memory precision tested. Subsequently, RT was measured as participants oriented to targets appearing in those
\end{abstract}

\section{INTRODUCTION}

Previous literature suggests that there are functional consequences of social distraction on learning and spatial contextual memory (Doherty, Patai, Duta, Nobre, \& Scerif, 2017). Both behavioral and gaze differences have been reported previously during visual search with naturalistic social and nonsocial scenes that were indicative of social distraction, and these behavioral differences were associated with subsequent poorer memory performance for target locations in scenes with social distractors. However, in addition to attention affecting memory performance, memory performance also affects attentional orienting, a bidirectional relationship that is well documented for both short-term (Kuhl \& Chun, 2014; Astle \& Scerif, 2011; Griffin \& Nobre, 2003) and longer-term memory (Goldfarb, Chun, \& Phelps, 2016; Rosen, Stern, Michalka, Devaney, \& Somers, 2016; Hutchinson \& TurkBrowne, 2012; Chun \& Turk-Browne, 2007). It is possible, therefore, that the effect of social distraction on learning and memory may, in turn, alter later orienting within natural scenes, completing this bidirectional chain. We investigated this novel memory-guided attentional orienting

\footnotetext{
${ }^{1}$ University of Oxford, ${ }^{2}$ University College London, ${ }^{3}$ University of California, San Francisco
}

scenes at either valid (previously learned) locations or invalid (different) locations. Memory precision was poorer for target locations in social scenes. In addition, distractor type moderated the validity effect during memory-guided attentional orienting, with a larger cost in RT when targets appeared at invalid (different) locations within scenes with social distractors. The poorer memory performance was also marked by reduced anticipatory dynamics of spatially lateralized 8-12 Hz alpha-band oscillations for scenes with social distractors. The functional consequences of a social attention bias therefore extend from memory to memory-guided attention orienting, a bidirectional chain that may further reinforce attentional biases. aspect and also asked whether the presence of social distractors during learning alters anticipatory neural dynamics during subsequent attentional orienting using EEG.

There is ample evidence that learning and memory guides attentional orienting. For example, the contextual cueing literature suggests that implicit memories for simple visual search arrays that have been seen previously reduce visual search time compared with novel search arrays (Chun \& Jiang, 1998). Other more recent work has built on these studies by reporting that spatial, contextual long-term memories for objects located within natural scenes also enhance perceptual sensitivity and RT during subsequent attention-orienting tasks (Doallo, Patai, \& Nobre, 2013; Patai, Buckley, \& Nobre, 2013; Patai, Doallo, \& Nobre, 2012; Stokes, Atherton, Patai, \& Nobre, 2012; Summerfield, Rao, Garside, \& Nobre, 2011; Summerfield, Lepsien, Gitelman, Mesulam, \& Nobre, 2006). In these tasks, participants search for target objects in natural scenes over several blocks to form a memory for where the target is located in each scene. Memory precision for target location is also tested (similar to Doherty et al., 2017). After a break, participants engage in an attentional orienting task. In one version, participants must react to the onset of the target superimposed on a previously studied scene in either the learned location (valid trials) or in a different location (invalid trials) while fixating 
centrally (Salvato, Patai, McCloud, \& Nobre, 2016; Salvato, Patai, \& Nobre, 2016; Summerfield et al., 2006). This memory-based Posner-style cueing task leads to a validity effect, whereby RT for valid trials is significantly faster than for invalid trials. This additional orienting task therefore allows for investigating the effects of memory on attention orienting. Hence, it can be classified as a memory-guided orienting task akin to contextual cueing. As social distraction affects memory performance, is it possible that this memory difference will carry over to subsequently affect attentional orienting.

In addition, can the effects of social distraction on memory-guided attention orienting be seen in neural activity? In the current study, we utilized natural social and nonsocial distractors embedded within scenes-human figures or objects with similar physical salience. Are there neural markers of memory-guided orienting of anticipatory attention that might be influenced by the nature of distractors within natural scenes? To address this question, we focused on anticipatory spatial lateralization of alpha-band oscillations, a neural marker of spatial attention that is well documented following both explicit symbolic cues (Haegens, Händel, \& Jensen, 2011; van Ede, de Lange, Jensen, \& Maris, 2011; Romei, Gross, \& Thut, 2010; Wyart \& Tallon-Baudry, 2008; Thut, Nietzel, Brandt, \& Pascual-Leone, 2006; Worden, Foxe, Wang, \& Simpson, 2000) as well as mnemonic cues for memoryguided orienting in natural scenes (Stokes et al., 2012; Summerfield et al., 2011). This phenomenon is generally thought to reflect relative up-regulation of neuronal excitability in task-relevant (contralateral) versus irrelevant (ipsilateral) neural populations ahead of anticipated perceptual targets (Stokes et al., 2012; Jensen \& Mazaheri, 2010; Kelly, Lalor, Reilly, \& Foxe, 2006) and is associated with improved perceptual performance (van Ede, de Lange, \& Maris, 2012; van Ede, Köster, \& Maris, 2012; Romei et al., 2010; van Dijk, Schoffelen, Oostenveld, \& Jensen, 2008; Thut et al., 2006). However, this spatial lateralization of alpha-band oscillations has not, to our knowledge, been studied in the context of natural scenes containing salient social distractors.

The current study therefore addressed two aims. The first aim was to explore the functional consequences of social distraction during learning on behavioral markers of attention: Will poorer explicit memory for target locations in social scenes after a visual search task affect memory-guided attention orienting in a Posner-like cueing task? The second aim of the current study was to identify a possible neural marker for the processing of natural scenes containing social distractors during memoryguided attention orienting. What are the neural consequences of social distraction on spatially lateralized memory-related alpha-band preparatory activity? Will poorer memory performance for social compared with nonsocial scenes also be reflected in attenuated preparatory alpha lateralization relative to the memorized location of anticipated perceptual target?

\section{METHODS}

Task design, data processing, statistical analyses, and task measures are the same as those used in the previous study (Doherty et al., 2017), unless otherwise noted.

\section{Participants}

The University of Oxford Central University Research Ethics Committee approved this research. Twenty healthy adult volunteers participated. All had normal or corrected-to-normal vision. Two participants were excluded because of inability to record EEG (due to particularly thick hair styling). The final sample consisted of 18 participants aged 19-21 years, of which 15 were women. Participants were recruited through a lab practical for undergraduate psychology students at the University of Oxford. All participants provided written informed consent and received course credit for their participation.

\section{Stimuli}

Stimuli were social and nonsocial distracters in 80 natural scenes equated for low-level visual salience, as established using a bottom-up visual saliency algorithm based on the original Itti and Koch algorithm (Harel, Koch, \& Perona, 2006). These scenes included various indoor and outdoor scenes prepared from photographs. Target objects were also photographs, including tools, toys, fruits, and so forth. Every scene had a unique target object placed within it. Matching social and nonsocial versions of each scene were created by editing a photograph of a social distractor (person) or nonsocial distractor into the same location in the scene. Stimuli were counterbalanced across participants similar to the previous study (Doherty et al., 2017), such that half of the participants saw the same 40 scenes as social and the other 40 as nonsocial, whereas the other half of participants saw the reverse. Also, there were two target locations in each scene (on the same side and the opposite side of the distractor) that was counterbalanced across participants. In addition to utilizing this previous counterbalancing scheme, the validity of the scene during the orienting phase (described below) was also counterbalanced. Although half of the participants saw the same 40 scenes as valid and the other 40 scenes as invalid, the other half saw the reverse. Within this counterbalancing, distractor location (left or right side) and sex of social distractors (male or female) were also balanced to the extent possible (see supplementary online material https://osf.io/hm5cs/). Visual salience with regard to low-level visual properties (color, contrast, etc.) was investigating using a bottom-up visual saliency algorithm (Harel et al., 2006), which demonstrated no significant differences in salience between isolated social and nonsocial distractors within scenes, as well as social and nonsocial scenes overall (see Doherty et al., 2017, for further details). 


\section{Procedure}

\section{Visual Search/Learning Phase}

Participants sat $60 \mathrm{~cm}$ away from a 23-in. monitor with a $1920 \times 1080$ resolution and spanning approximately $46 \times 27$ degrees of visual angle. Participants were directed to look for target objects in 80 scenes over three blocks. For each trial, participants saw (1) a fixation square for 1000-1500 msec, (2) the object alone for $3000 \mathrm{msec}$, (3) the scene and embedded object until they located the scene or the time expired, and (4) feedback for 1000 msec ("Great job!" or "Uh oh!"). Maximum search time was $20 \mathrm{sec}$ in the first block and decreased by $4 \mathrm{sec}$ in each subsequent block. Participants observed all 80 scenes in random order during each of three blocks, and each scene had a unique target object.

Several small adjustments were introduced compared with a previous study (Doherty et al., 2017). Instead of pressing the spacebar to reveal the cursor once they located the target objects, participants pressed the mouse. Because a higher resolution monitor was used for this task, the targets and scenes were displayed centrally using the resolution of the previous study (central object: $2.01 \times 2.01$ degrees of visual angle; scenes: $45.45 \times$ 26.73 degrees of visual angle; target in scene: $1.36 \times$ 1.36 degrees of visual angle) with a gray $(153,153,153)$ border surrounding. A practice phase was included before the task, consisting of 12 trials. The task was cartoonthemed, including images of characters during the feedback and instruction screens, a story for the task including the characters to make the task appear more like a game, and points acquired after each block. These points were random and increasing from block to block, to keep participants motivated and not discouraged in subsequent developmental experiments including child participants. When the targets were correctly located, they flashed bigger and smaller for positive reinforcement. These last three alterations were included to make the task child-friendly for compatibility with subsequent developmental experiments.

The current study used a Tobii TX300 eye tracker with gaze recorded from both eyes at $300 \mathrm{~Hz}$ following a 9-point calibration. Participants' eye gaze positions were calibrated before the start of each block.

\section{Memory Phase}

After a short break, explicit memory for target locations was probed. This phase differed from the previous study (Doherty et al., 2017) by including a cartoon-themed introduction. Each of the studied scenes was presented in a random order with its accompanying social/nonsocial distracter. Participants moved the cursor shaped as the target for that scene and click when the target was in the location they remembered it to be in the scene.

\section{Orienting Phase}

After the memory phase, participants were capped for EEG, which lasted approximately $30 \mathrm{~min}$. Participants then engaged in a memory-guided orienting task in which they reacted to the brief appearance of targets within their associated scenes while holding their gaze at a centrally located fixation cross present during the entire presentation of scenes.

Trials commenced with the presentation of the central target $(2.16 \times 2.16$ degrees of visual angle) for $3000 \mathrm{msec}$ (Figure 1). A fixation cross on a blank screen followed for 500-1500 msec, after which the associated scene appeared. The target object was presented superimposed on the scene for $100 \mathrm{msec}$ after 1000-1500 msec. After

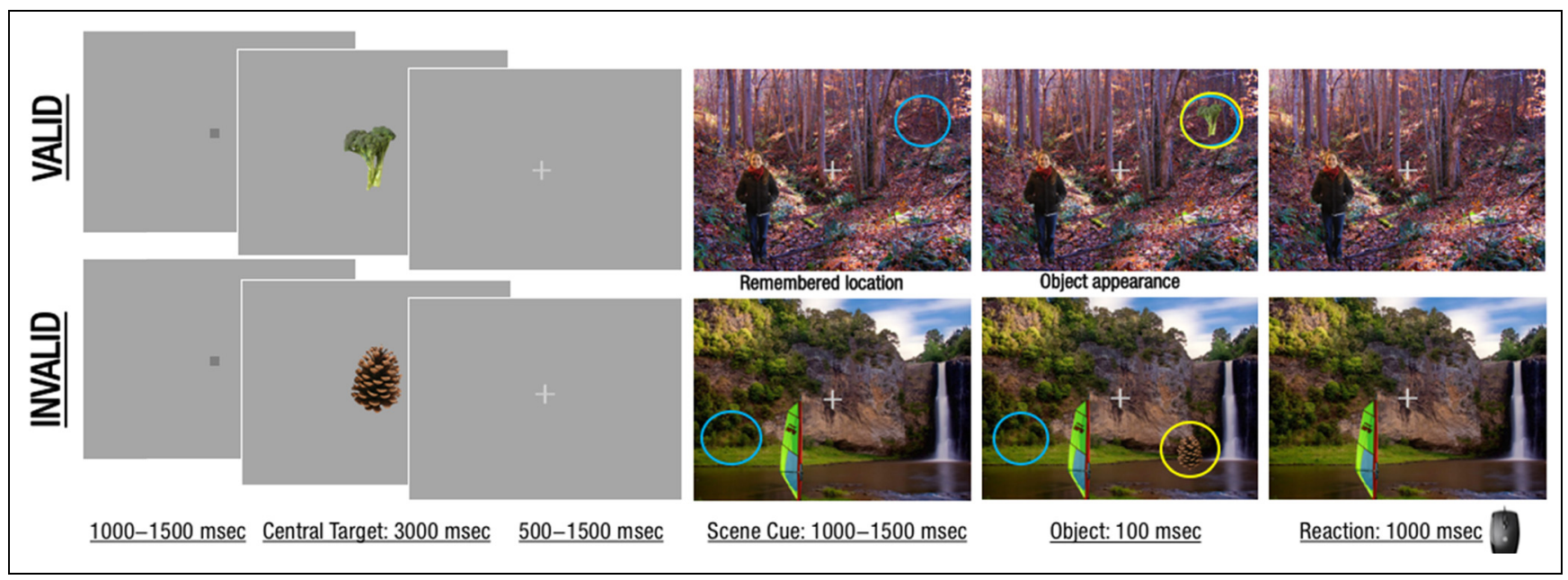

Figure 1. Trial sequence for the orienting phase. Participants viewed (A) a fixation square, (B) the centrally presented target, (C) a warning cross, (D) the scene, (E) the target appearance, and (F) RT window. Blue circles are examples of the remembered location for the target, and yellow circles are examples of the location of the target appearance during the orienting phase. These circles were not present during the task and are for illustrative purposes. Targets have been magnified for illustrative purposes. 
target disappearance, the scene remained present for 1000 msec, providing a response window. A fixation point remained present throughout the trial. After each trial, a blank screen appeared for $1500 \mathrm{msec}$, during which participants could blink, followed by the presentation of a fixation square lasting between 1000 and $1500 \mathrm{msec}$, which prompted participants to get ready for the next trial. Participants responded to the presentation of the target object by pressing the left mouse button if the target appeared on the left and the right mouse button if the target appeared on the right. Participants observed all 80 scenes in random order. Although previous studies often utilized a present/absent discrimination after entire scenes were presented briefly (200 msec; e.g., Patai et al., 2012), this version was chosen for being simpler for use with children in subsequent experiments. In half the scenes, the object appeared in the learned location (valid trials), and in the other half, the object appeared in a new location in the opposite hemifield (invalid trials).

At the start of EEG testing, participants were instructed to relax and move their heads as little as possible. In addition, they were asked to minimize blinking and to avoid making saccades during experimental trials. Participants were then allowed 12 practice trials to make sure they understood the task. To encourage fewer blinks during each trial, participants were instructed to blink during the blank screen that preceded the appearance of the fixation square. To reduce fatigue, participants had a short break every 16 trials. The entire orienting task, including practice trials, lasted approximately $15 \mathrm{~min}$.

\section{EEG Acquisition and Processing}

EEG activity was recorded using a 128-channel Hydrocel Geodesic Sensor Net connected to Net Amps 300 (Electrical Geodesics, Inc.) using NetStation 4.5 software. EEG signal was referenced online to the vertex and was sampled at $250 \mathrm{~Hz}$. Electrode impedances were kept below $5 \mathrm{k} \Omega$ as recommended by the manufacturer. Eye movements and eye blinks were monitored with six eye channels placed on the outer canthi of both eyes and above and below the eyes. EEG processing was conducted using Fieldtrip software (Oostenveld, Fries, Maris, \& Schoffelen, 2011). EEG data were rereferenced the average reference, demeaned, and segmented into three time epochs offline.

Three types of epochs were segmented from the continuous EEG stream to analyze oscillatory activity induced by the central object cue (epoch indicated by Figure 1A), the scene (epoch indicated by Figure 1D), and the target appearance (epoch indicated by Figure 1E). The segments were as follows: (1) $500 \mathrm{msec}$ before and $4000 \mathrm{msec}$ after central object presentation, (2) $2000 \mathrm{msec}$ before and $2000 \mathrm{msec}$ after scene presentation, and (3) $1000 \mathrm{msec}$ before and $2000 \mathrm{msec}$ after target presentation. Fieldtrip's fast independent component analysis (ICA) was used for removing the artifactual independent components associated with ocular artifacts (blinks and eye movements) from the data while keeping trials in the analyses. To find the noise components of interest, we correlated component time courses with the measured vertical and horizontal EOG signals. Trials with muscle artifacts were removed prior to ICA. This exclusion of trials resulted in an average of 38.94 nonsocial trials $(S D=$ $1.51)$ and 39.11 social trials $(S D=1.23)$ included in the EEG analyses per person for the central object epoch. For the scene epoch, this resulted in an average of 39.11 nonsocial trials $(S D=1.13)$ and 38.78 social trials $(S D=1.17)$ per person. Finally, 39.33 nonsocial trials $(S D=0.84)$ and 39.37 social trials $(S D=0.92)$ were included in the target appearance EEG analyses. Bad channels, identified via visual inspection, were removed and replaced by interpolated values. To investigate transient neural dynamics following the brief target presentation, a time-frequency analysis was performed using a shorttime Fourier transform in combination with a Hanning taper. We used a sliding time window of $500 \mathrm{msec}$ that was advanced over the data in $25-\mathrm{msec}$ steps. These data were primarily used to find the channels of interest that showed the maximal difference in the posttarget alpha response following left versus right visual targets. In contrast to this brief target response, we predicted the anticipatory modulation of oscillatory activity in response to the central object and the scene to be much more sustained and therefore applied a sliding time window of $1000 \mathrm{msec}$ (in steps of $50 \mathrm{msec}$ ) instead. To increase sensitivity further, this larger time window also enabled us to utilize multitapering of the data (Percival \& Walden, 1993) to achieve $\pm 3 \mathrm{~Hz}$ smoothing, as this would be beneficial in averaging across participants with slightly different peak frequencies.

\section{Eye Tracking}

Eye-tracking data from the left eye were processed and analyzed using custom MATLAB scripts. Gaze data were first preprocessed. Trials were determined invalid if 1000 msec of consecutive gaze points were invalid or if more than $40 \%$ of gaze points were invalid during the entire trial. Trials with invalid data were removed from the analysis. Periods of blink were replaced with the last good values. Fixations were calculated using a maximum velocity threshold of 75 degrees of visual angle/sec, a dispersion threshold of 0.5 degrees of visual angle around the fixation centroid and a minimum duration threshold of $50 \mathrm{msec}$. Areas of interest were hand drawn around distracters.

\section{Statistical Analysis}

\section{Visual Search/Learning Phase}

Search time from scene onset to click on target (sec) and first look (whether the first saccade and associated fixation after scene onset was to the distractor) were both 
calculated only for trials in which the target was accurately located. Accuracy was calculated as whether participants correctly clicked on the target within a buffer of 0.63 degrees of visual angle.

\section{Memory Phase}

Memory error was measured as distance in pixels from the accurate target location to recalled location, for trials in which participants accurately found the target object at least once in the visual search task.

\section{Orienting Phase}

Accuracy was calculated as trials in which participants correctly responded to the location of the target appearance (on the left or right hemisphere) with the left or right mouse button within the RT window. RT from target onset to mouse press was calculated for accurate trials. Trials included for RT analyses were also limited to trials in which participants accurately found the target object at least once during the visual search task, and trials in which the RT was within two standard deviations of the mean for that condition for that participant.

\section{Akaike Information Criterion Modeling}

For each dependent measure, model averaging was performed. An information-theoretic approach using Akaike information criterion (AIC) modeling (Burnham \& Anderson, 2002) was used for statistical analysis. In this approach, a global linear mixed-effects model was first created using all fixed predictor variables of interest, with subject and scene as random variables to account for the nonindependence across trials within subjects and across blocks of the visual search task within scenes. Random slopes were included in the mixed-effects models according to the "best path" method described in the literature (Barr, Levy, Scheepers, \& Tily, 2013). Next, a subset of candidate models that contained all possible combina- tions of the fixed effects included in the global model was specified. Finally, Akaike weight-based averaging over all candidate models allowed for the derivation of mean estimates of the coefficients $(\theta)$ as well as 95\% confidence intervals (CIs), to be used for determining which coefficients were statistically significantly different from zero. This approach allowed us to include all trials in the analyses as opposed to averaging over trials, which incorporates the variance within subjects into the model. It also allowed us to analyze proportion data more appropriately by using logit linear mixed-effects models (generalized linear mixed-effects models for binomially distributed outcomes) with the binary response variable first look (Jaeger, 2008). All models were checked for the assumptions of normality and homogeneity of variance using visual inspection in $\mathrm{R}$. If data were not normally distributed, data were transformed with natural log (ln). The variable search time was the only variable transformed and was done so due to positive skew.

\section{EEG Analyses}

Contrasts were created to investigate lateralized activity (contralateral-ipsilateral) to the learned target location (for central object and scene epochs) and the target presentation (target appearance epoch). To focus the analysis on the electrodes that were relevant to target processing, while also ensuring that data selection was independent of the analysis of interest, left and right channels of interest were selected on the basis of the post-target-induced alpha response. These channels were then used to zoom in on the effect of interest in the pretarget anticipatory intervals (following central target cue and scene onsets). To deal with variability across frequencies (1/f) and participants, we normalized these contraipsi differences to a percentage change by dividing them by their sum and multiplying the resulting value by 100 (i.e., $([$ contra - ipsi $] /[$ contra + ipsi $]) \times 100)$. To deal with the multiple comparisons encountered along the time and frequency axes, we relied on cluster-based
Figure 2. Mean proportion of trials with first looks and search times for social and nonsocial scenes over three blocks during visual search. Error bars are SEMs. Participants made more first looks toward social distractors compared with nonsocial distractors in Block 1, but not in Blocks 2 and 3. Participants also had shallower search time slopes over three blocks for social scenes compared with nonsocial scenes.

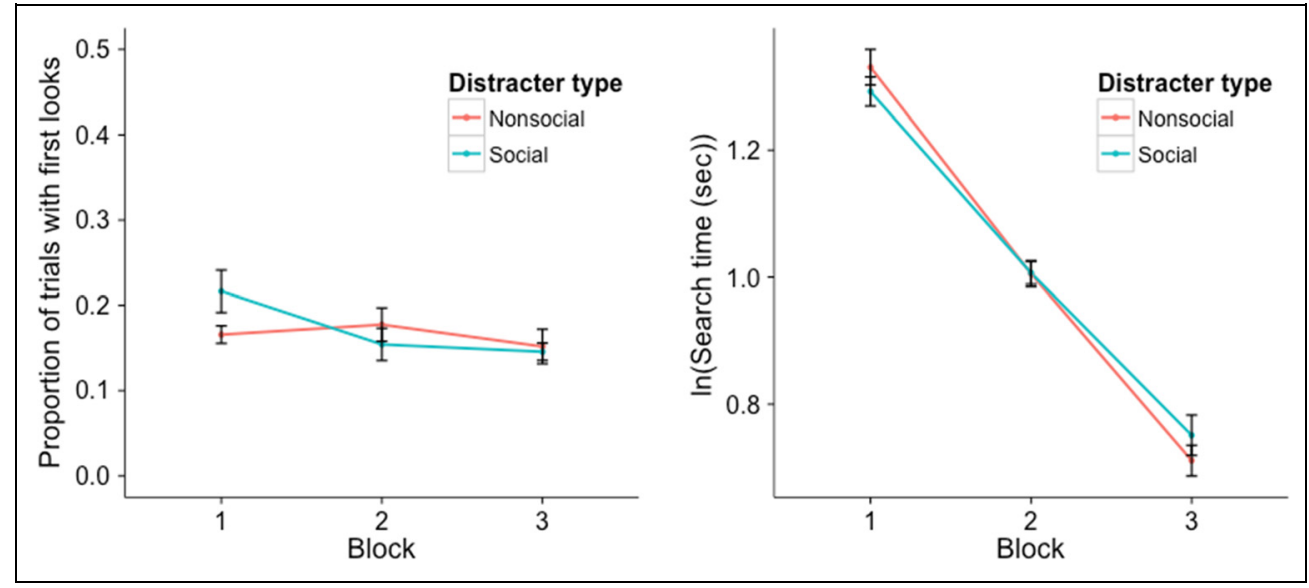


Table 1. Model Averaging with Parameters Related to the Dependent Measures during Visual Search

\begin{tabular}{|c|c|c|c|c|c|c|c|c|}
\hline \multirow[b]{2}{*}{ Predictor } & \multicolumn{4}{|c|}{ Search Time $(\ln (S))$} & \multicolumn{4}{|c|}{ First Look (Yes/No) } \\
\hline & Estimate & $l-95 \% C I$ & $u-95 \% C I$ & $p$ & Estimate & $l-95 \% C I$ & $u-95 \% C I$ & $p$ \\
\hline (Intercept) & 1.206 & 1.094 & 1.321 & $<.001$ & -1.818 & -2.157 & -1.475 & $<.001$ \\
\hline Block & -0.538 & -0.623 & -0.459 & $<.001$ & -0.195 & -0.528 & 0.138 & .251 \\
\hline Distractor & -0.015 & -0.061 & 0.012 & .495 & 0.26 & -0.225 & 0.761 & .305 \\
\hline Distractor $\times$ Block & 0.063 & 0.011 & 0.115 & .017 & -0.548 & -0.972 & -0.124 & .011 \\
\hline
\end{tabular}

For each parameter and dependent measure, this table presents the averaged coefficient estimates $(\theta \mathrm{s})$ and the $95 \%$ CIs $(\mathrm{l}=\mathrm{lower}, \mathrm{u}=\mathrm{upper})$ based on estimated unconditional variance. Estimates in bold differed statistically from zero based on $95 \%$ CIs with $p<.05$.

permutation tests (Maris \& Oostenveld, 2007) as implemented in the Fieldtrip software. In essence, this approach circumvents the multiple-comparison problem by evaluating the full data space (time-frequency space) under a single permutation distribution of the largest cluster. In essence, $[$ contra $-\mathrm{ipsi}] /$ [contra $+\mathrm{ipsi}]) \times$ 100 was compared against 0 in statistical tests, and this equates to (contra / [contra + ipsi]) vs. (ipsi / [contra + ipsi]). For cluster thresholding, we used the conventional univariate two-tailed alpha level of .05. To obtain cluster $p$ values, we permuted the data 1000 times.

\section{RESULTS}

\section{Visual Search/Learning Phase}

\section{Accuracy (\%)}

Accuracy was at ceiling, with no significant effects other than the intercept when using model averaging (results not shown; Social Block 1: $M=97.88 \%, S D=3.79 \%$; Social Block 2: $M=98.31 \%, S D=1.96 \%$; Social Block 3: $M=98.75 \%, S D=2.26 \%$; Nonsocial Block 1: $M=$ 97.78\%, $S D=2.43 \%$; Nonsocial Block 2: $M=98.06 \%$, $S D=2.24 \%$; Nonsocial Block $3: M=98.19 \%, S D=$ $2.32 \%)$.

\section{First Look (Yes/No)}

Because of the binary nature of the dependent measure (participants either made a fist look to the distracter in each trial or they did not), logit mixed-effects models (generalized linear mixed-effects models for binomially distributed outcomes) were used in AIC modeling. The only fixed-effect coefficient that was significantly different from zero was the interaction between distractor and block. Post hoc analyses with subject-averaged data revealed this interaction to be driven by a significantly higher proportion of first looks to social compared with nonsocial distractors in Block $1(p=.047)$, but no significant differences in Blocks 2 and $3(p>.250$; Figure 2$)$.

\section{Search Time (sec)}

AIC model averaging with search time during the visual search task revealed that the coefficient estimates for block as well as for the interaction between distractor and block were significantly different from zero, indicating their significant effects on the model (Table 1). The effect of block was driven by large reductions in search times as scene-target associations became learned over the course of the three blocks (see Figure 2). In light of the significant effect of the Distractor $\times$ Block interaction term in model averaging, investigating the slopes in a similar manner to the previous study (Doherty et al., 2017) revealed shallower search slopes for scenes with social distractors compared with nonsocial distractors (Figure 2).

To investigate whether gaze behavior toward distractors predicted search time during the visual search task, first look, distractor, and block, as well as all possible interactions between these variables, were entered as predictors for search time in AIC model averaging. Apart

Table 2. Model Averaging with Parameters Relevant for Orienting Phase RT (sec)

\begin{tabular}{lcccr}
\hline Predictor & Estimate & $l-95 \% C I$ & $u-95 \% C I$ & \multicolumn{1}{c}{$p$} \\
\hline Intercept) & 0.394 & 0.37 & 0.418 & $\mathbf{0}$ \\
Distractor & $\mathbf{0 . 0 1 5}$ & $\mathbf{0 . 0 0 5}$ & $\mathbf{0 . 0 2 6}$ & $\mathbf{0 1 2}$ \\
Validity & $\mathbf{- 0 . 0 3}$ & $\mathbf{- 0 . 0 4 1}$ & $\mathbf{- 0 . 0 1 8}$ & $<.001$ \\
Distractor $\times$ Validity & $\mathbf{- 0 . 0 2 1}$ & $\mathbf{- 0 . 0 3 6}$ & $\mathbf{- 0 . 0 0 6}$ & $\mathbf{0 0 6}$ \\
\hline
\end{tabular}

For each parameter, this table presents the averaged coefficient estimates $(\theta \mathrm{s})$ and the $95 \% \mathrm{CIs}(\mathrm{l}=$ lower, $\mathrm{u}=$ upper $)$ based on estimated unconditional variance. Estimates in bold differed statistically from zero based on 95\% CIs with $p<.05$. 
from the effects described above, there was only a nonsignificant trend for the interaction between first look and distractor $(p=.056)$, and no other effects including first look $(p>.250$; results not shown here).

\section{Memory Phase}

To determine whether social distraction during the learning phase translated into poorer subsequent memory for scenes social distractors (as in the previous study: Doherty et al., 2017), memory error (distance in pixels from recalled to correct location) was analyzed. AIC modeling revealed a significant effect of distractor type (estimate = $12.63, p=.028$, no CIs due to only having one fixed effect: distractor), with poorer memory (larger error) for scenes with social distractors (social: $M=185.57, S D=26.18$; nonsocial: $M=158.56, S D=26.18)$, similar to the previous study (Doherty et al., 2017).

\section{Orienting Phase}

Accuracy (\%)

Accuracy was at ceiling, with no effects other than a significant coefficient for the intercept (results not shown; social valid: $M=97.59 \%, S D=1.96 \%$; social invalid: $M=98.18 \%, S D=2.37 \%$; nonsocial valid: $M=$ 98.57\%, $S D=1.98 \%$; nonsocial invalid: $M=98.85 \%$, $S D=2.52 \%)$.

\section{$R T$ (sec)}

Model averaging using RT during the orienting phase showed a significant effect of all three fixed effects: distractor, validity, and the Distractor $\times$ Validity interaction (Table 2). The effect of validity was driven by longer RTs for invalid trials. The Distractor $\times$ Validity interaction was followed up with subject-averaged post hoc analyses, which revealed this interaction to be driven by signif-

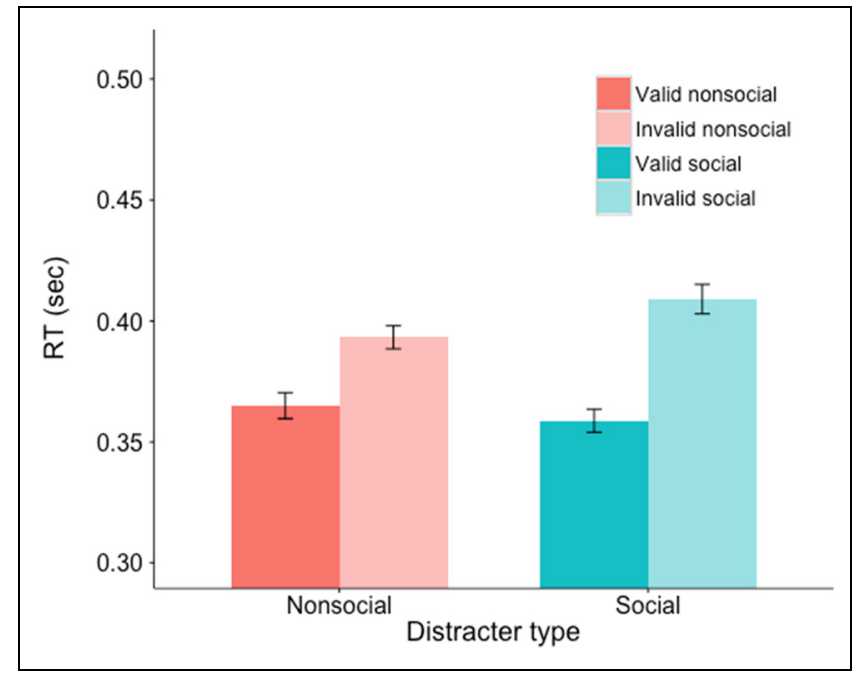

Figure 3. Mean RTs during the orienting phase demonstrate an overall validity effect for both social and nonsocial scenes, with slower RTs for invalid compared with valid trials, while also showing a moderating effect of distractor type with slower RTs for invalid social trials compared with invalid nonsocial trials. Error bars are SEMs.

icantly longer RTs for invalid social trials compared with invalid nonsocial trials $(p=.030)$, but no difference between social and nonsocial scenes for valid trials $(p>$ .250; Figure 3).

\section{Relationships among Learning, Memory, and Attention}

To investigate whether gaze behavior or search slope during the visual search task predicted memory error during the memory phase, search time slope (the regression slope of the search times across all three blocks for a particular scene), first look proportion (the proportion of the blocks out of three in which the participant made a first look at the distractor for a particular scene), and

Table 3. Visual Search Measures (Search Time Slope and First Look Proportion) as Predictors for Memory Error during the Memory Phase

\begin{tabular}{lccc}
\hline Predictor & Estimate & $l-95 \% C I$ & $u$-95\% CI \\
\hline Intercept) & 154.591 & 110.313 & 197.809 \\
Search time slope & $\mathbf{2 5 . 6 5 4}$ & $\mathbf{4 . 0 9 8}$ & $\mathbf{4 6 . 7 9 8}$ \\
Distractor & $\mathbf{3 1 . 1 8 6}$ & $\mathbf{3 . 2 4 6}$ & $\mathbf{5 9 . 1 2 6}$ \\
Distractor $\times$ Search Time Slope & 23.717 & -4.624 & $\mathbf{. 0 1 9}$ \\
First look proportion & 12.339 & -6.441 & $\mathbf{. 0 2 9}$ \\
Distractor $\times$ First Look Proportion & -17.81 & -46.367 & 31.247 \\
First Look Proportion $\times$ Search Time Slope & -2.743 & -16.465 & 10.704 \\
First Look Proportion $\times$ Search Time Slope $\times$ Distractor & -5.887 & -32.927 & .101 \\
\hline
\end{tabular}

For each parameter, this table presents the averaged coefficient estimates $(\theta \mathrm{s})$ and the $95 \%$ CIs $(1=10 w e r$, $\mathrm{u}=\mathrm{upper})$ based on estimated unconditional variance. Estimates in bold differed statistically from zero based on 95\% CIs with $p<.05$. 
Table 4. Memory Error (Pixels) during the Memory Phase as a Predictor for Orienting Phase RT

\begin{tabular}{|c|c|c|c|c|}
\hline Predictor & Estimate & $l-95 \% C I$ & $u-95 \% C I$ & $p$ \\
\hline (Intercept) & 0.395 & 0.371 & 0.418 & $<.001$ \\
\hline Memory error & -0.003 & -0.011 & 0.005 & .538 \\
\hline Distractor & 0.016 & 0.005 & 0.027 & .01 \\
\hline Validity & -0.03 & -0.041 & -0.018 & $<.001$ \\
\hline Memory Error $\times$ Distractor & -0.007 & -0.017 & 0.004 & .199 \\
\hline Memory Error $\times$ Validity & 0.015 & 0.004 & 0.025 & .006 \\
\hline Distractor $\times$ Validity & -0.025 & -0.042 & -0.009 & .002 \\
\hline Memory Error $\times$ Distractor $\times$ Validity & -0.007 & -0.024 & 0.01 & .426 \\
\hline
\end{tabular}

For each parameter, this table presents the averaged coefficient estimates $(\theta \mathrm{s})$ and the $95 \% \mathrm{CIs}(\mathrm{l}=$ lower, $\mathrm{u}=$ upper $)$ based on estimated unconditional variance. Estimates in bold differed statistically from zero based on 95\% CIs with $p<.05$.

distractor were entered as predictors for memory error in model averaging. In addition to the effects seen in the above memory phase analyses, there was a significant effect of search time slope, with shallower slopes associated with poorer memory precision (higher error), similar to participants in the previous study (Doherty et al., 2017; Table 3).

To investigate whether memory precision during the memory phase subsequently predicted RTs in the orienting phase, memory error, distractor, validity, and all interactions between these variables were entered as fixed effects in AIC model averaging. In addition to the effects described above, there was also a significant effect on the model of the interaction between memory error and validity (Table 4). Following up on this interaction, post hoc analyses with subject averages revealed that there was a significant positive correlation between memory error and RT for valid trials during orienting $(r=.52$, $p=.027$ ), with worse memory precision related to longer RTs. In contrast, there was no significant correlation for invalid trials $(r=.15, p>.250$; Figure 4$)$.

\section{EEG Analyses}

\section{Alpha Desynchronization}

We used the alpha-band response induced by left and right targets to find the channels of interest, which could then subsequently be used to look at pretarget (anticipatory) modulations by spatial orienting following central target cue and scene onsets. The topography for alpha activity (8-12 Hz) contralateral compared with ipsilateral to the target location was inspected from 100 to $600 \mathrm{msec}$ poststimulus. Alpha suppression (desynchronization) in contralateral (relative to ipsilateral) targets was most evident in the posterior channels highlighted in Figure 5. Inspecting the time-frequency plot for the contralateral-ipsilateral target contrast, averaged over these highlighted channels, showed that this target response was most prominent between 200 and 400 msec posttarget (Figure 5).

Contralateral-ipsilateral contrasts averaged over the highlighted posterior channels were then used to investigate preparatory alpha lateralization following the central object cue as well as the scene presentation. Associated time-frequency contrasts were then evaluated using cluster-based permutation tests.

As we expected given the prior literature on memoryguided preparatory attention, for centrally presented targets associated with scenes with nonsocial distractors, we found a significant cluster when comparing power contralateral compared with ipsilateral that encompassed the alpha range, $p=.014$ (Figure 6). In contrast, there were no detectable significant differences in power contralateral compared with ipsilateral in response to

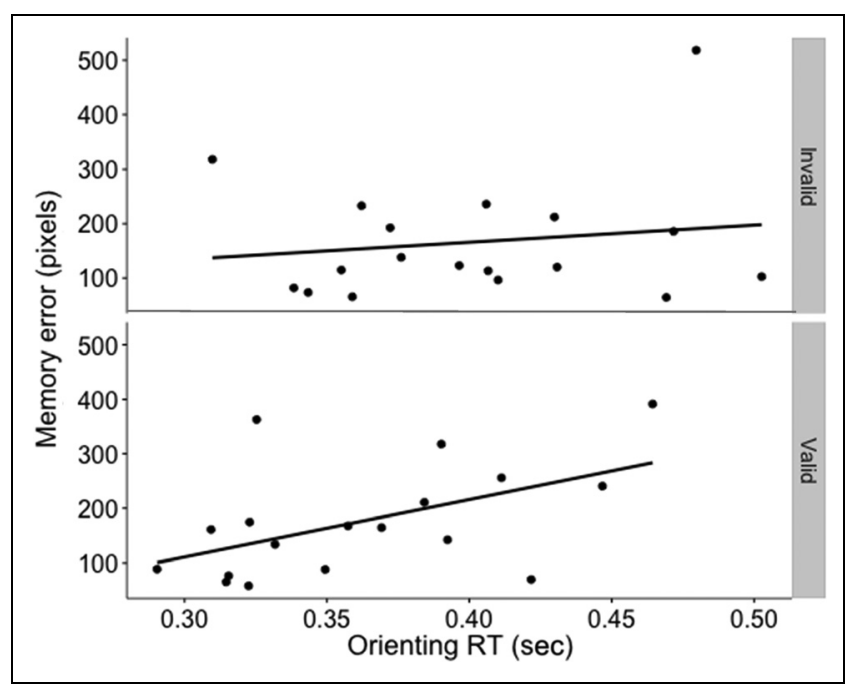

Figure 4. Depicts the significant relationship between memory error (pixels) during the memory phase and RT (sec) during the orienting phase for subject averages for valid trials, but no significant relationship for invalid trials. Results still hold without the potential outlier in invalid trials. 
Figure 5. The topography for alpha activity $(8-12 \mathrm{~Hz})$ contralateral compared with ipsilateral to the location the target briefly appeared on the screen averaged from 100 to 600 msec poststimulus, with posterior channels used for follow-up analyses highlighted (left), and time-frequency plot for contralateral-ipsilateral percent power change averaged over these highlighted symmetrical channel pairs (right).

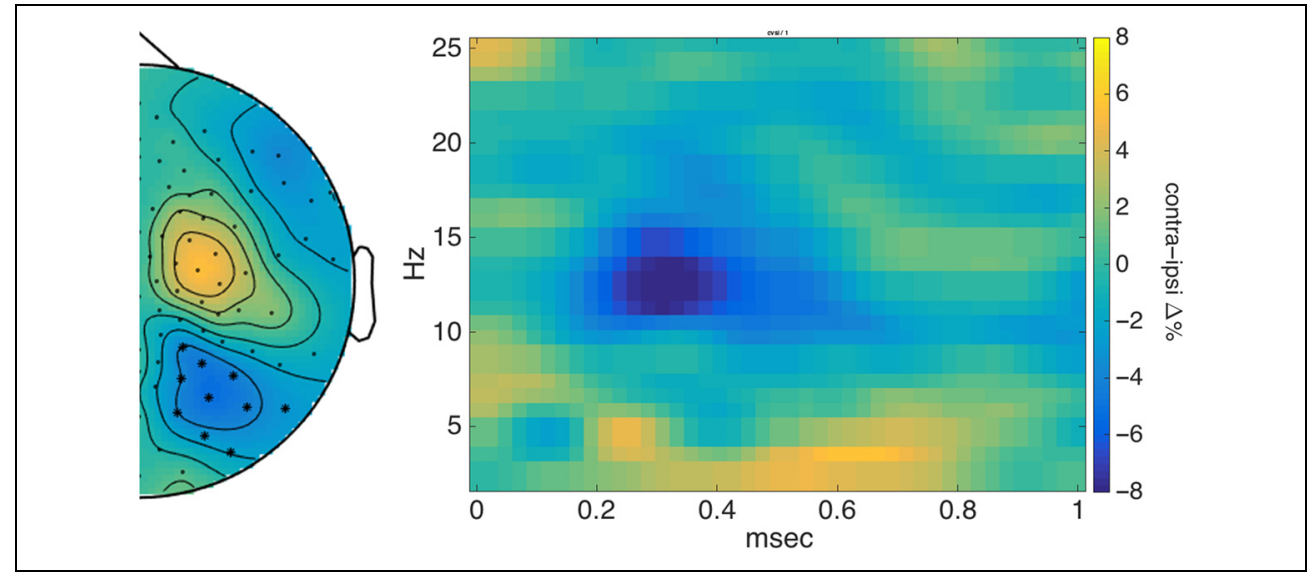

centrally presented targets associated with scenes with social distractors. To follow up the significant cluster for centrally presented targets associated with nonsocial scenes, a cluster-based permutation test over time was conducted with contrasts averaged over the alpha range
(8-12 Hz) specifically. Although there were no significant clusters in response to the presentation of central objects associated with scenes with either social or nonsocial distractors, there was a nonsignificant trend for nonsocial scenes $(p=.050)$. At face value, this contrasts with the
Figure 6. (A) Contralateralipsilateral percent power change in oscillatory activity over time in response to the central object (left) and the scene presentation (right) for social and nonsocial associated targets and scenes, extracted from the posterior channels highlighted in Figure 5.

(B) Contralateral-ipsilateral percent power change in oscillatory activity over time for alpha power specifically $(8-12 \mathrm{~Hz})$, cluster outline indicates significant decrease for contralateral versus ipsilateral for nonsocial during scene presentation. (C) Topographies for contralateral-ipsilateral percent power change in alpha (8-12 Hz) oscillations averaged over 500 msec time segments (C).

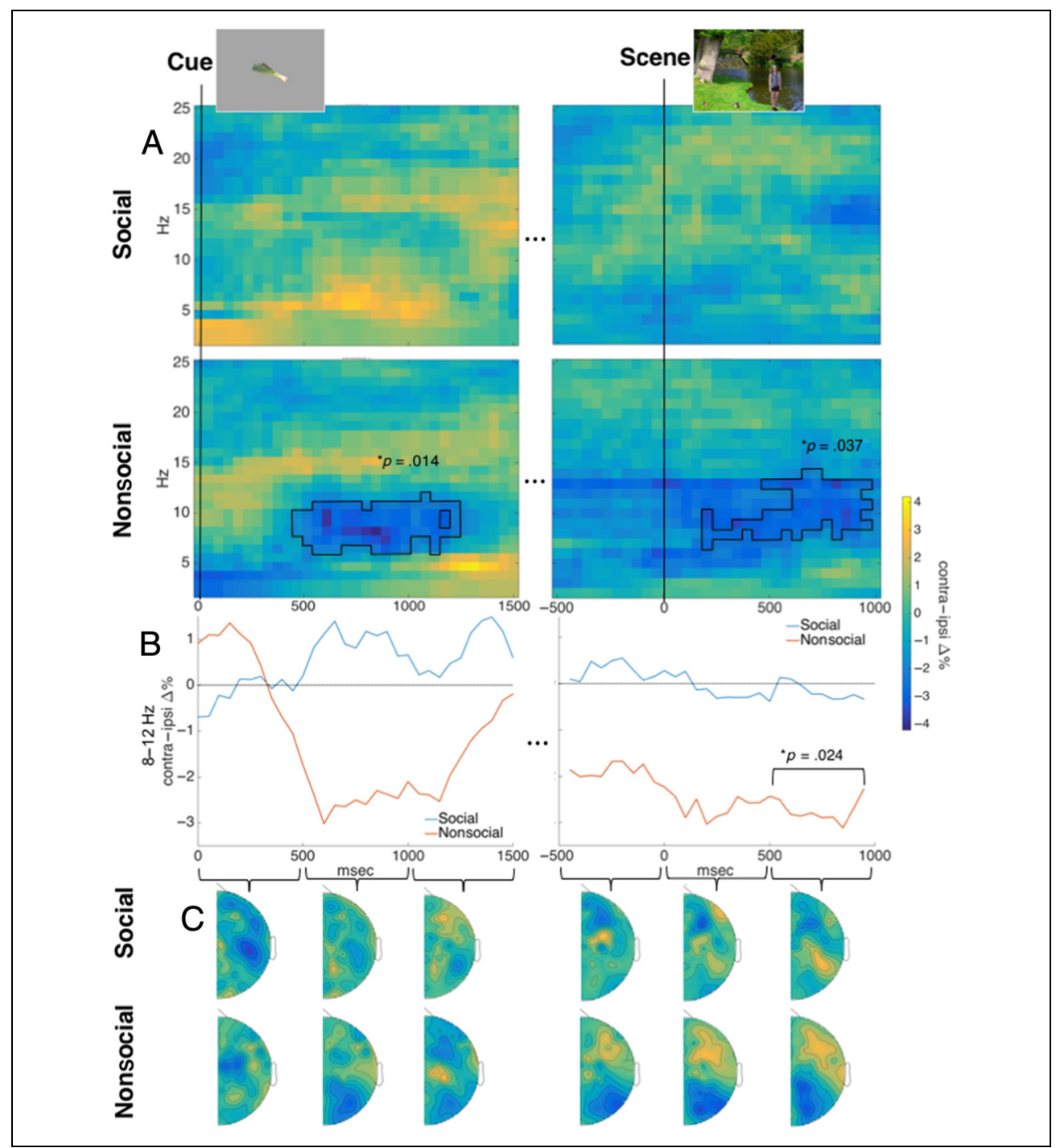


cluster observed in the time-frequency evaluation. However, careful examination of the time-frequency plot suggests this may be due to the fact that this desynchronization cluster following object cues peaks in slightly lower frequencies than typical considered for alpha oscillations. Examining the topographies of the difference between contralateral and ipsilateral alpha power $(8-12 \mathrm{~Hz})$ over time confirmed a largely posterior localization of these results and is, again, most clearly evident in the nonsocial case (Figure 6). There were no significant differences when comparing contralateral-ipsilateral contrasts between scenes with social and nonsocial distractors directly $(p=.11)$.

For the scene presentation, we found a significant cluster when comparing power contralateral compared with ipsilateral that encompassed the alpha range in response to scenes with nonsocial distractors, $p=.037$ (Figure 6). In contrast, there were no detectable significant differences in power contralateral compared with ipsilateral in response to the presentation of scenes with social distractors. To follow up the significant cluster for nonsocial scenes, a cluster-based permutation test over time was conducted with contrasts averaged over the alpha range $(8-12 \mathrm{~Hz})$ specifically. A significant negative cluster was detected in response to scene presentation for scenes associated with nonsocial targets from 500 to $1000 \mathrm{msec}$, indicating less power (desynchronization) contralateral compared with ipsilateral to the side of the remembered target location (Figure 6). Again, there were no significant differences when comparing contralateral-ipsilateral contrasts between scenes with social and nonsocial distractors directly $(p>.250)$.

As a very helpful reviewer noted, preparatory alphaband lateralization for targets associated with nonsocial scenes and alpha modulation for nonsocial scenes themselves in the absence of a similar modulation for targets and social scenes provide converging but weak, statistical evidence for an effect of social distraction on these neural markers. This is because the laterality effects in the nonsocial condition but not the social condition were not supported by the two corresponding statistically significant interaction effects of hemisphere (contralateral, ipsilateral) and scene (social, nonsocial). In turn, the absence of interaction effects is problematic (Nieuwenhuis, Forstmann, \& Wagenmakers, 2011), as at best we can speak about absent lateralization in the social condition, not a difference between social and nonsocial conditions. Although we believe that this is a very important issue and one that points to the need to replicate our alphaband findings, we would like to point to the qualitative absence of modulation for social scenes, as graphically shown in Figure 6A and B.

\section{DISCUSSION}

The current study built on the previous findings of the effects of social distraction on learning and subsequent memory for natural scenes (Doherty et al., 2017) to investigate the behavioral consequences of social distraction on subsequent memory-guided orienting of attention as well as on anticipatory neural dynamics during said memory-guided attentional orienting. Importantly, we replicated the effect of social distraction on subsequent memory, with poorer explicit memory for targets in scenes with social distractors. Interestingly and contrary to our original hypothesis, this did not attenuate memorybased orienting. In contrast, memory-guided attention orienting, as measured by the magnitude of the validity effect, was boosted for scenes previously associated with social distractors, with a greater cost for invalid trials in particular. EEG measures revealed an effect of preparatory attention on lateralized alpha power, typically considered a marker of spatial attention, for targets associated with nonsocial scenes: significant alpha $(8-12 \mathrm{~Hz})$ lateralization occurred after presentation of central targets associated with scenes with nonsocial distractors and of the scenes themselves. This finding is consistent with prior work and suggestive of changes in excitability in posterior cortical regions in anticipation for targets appearing at learned location, although likely reflects a mix of post-cue mnemonic retrieval and pretarget anticipatory dynamics. Alpha lateralization was absent for scenes containing social distractors. These neural findings require replication, as we did not find an interaction effect between laterality and scene type, but they converge with our behavioral data and they suggest a possible knock-on effect of an attention bias towards social stimuli. The presence of social stimuli in natural scenes, even when such stimuli are task irrelevant, results in poorer explicit contextual memory, which subsequently affects memoryguided attention orienting at both behavioral and possibly neural levels.

Important to note is the fact that the current study replicated the main results from the previous study (Doherty et al., 2017). First, we replicated here the difference in search time slopes between social and nonsocial scenes during the visual search task, with shallower slopes over three blocks for social scenes. This subtle difference was subsequently followed by a more pronounced difference in memory precision, with poorer memory for scenes containing social distractors, similar to the previous study. Finally, we replicated the cross-task relationship whereby search slope during visual search predicted memory precision in the memory phase, with shallower search slopes relating to poorer precision. With regard to first look, there was a subtle difference in the results between the two studies. Whereas participants in the previous study made more first looks to social distractors compared with nonsocial distractors across all three blocks, participants in the current study only made more first looks to social distractors in the first block. Given this discrepancy, it is possible that, although participants may consistently demonstrate social distraction in their search times and memory precision in this 
task, there is more variability in the degree of overt attention capture as measured by gaze behavior.

One intriguing finding from the current study is the fact that, although we show "poorer" explicit memory for scenes containing social compared with nonsocial distractors, we show a "greater" validity effect with a higher cost (slower RTs) for invalid social trials in particular. One explanation may lie in the cross-task analyses. We found that memory precision interacted with validity to predict RTs during the orienting phase, such that poorer memory precision related to longer RTs in valid trials; however, there was no such relationship for invalid trials. As the distractor effect at the orienting phase was driven by "invalid" trials, this cross-task analysis suggests that something other than explicit memory may be driving differential orienting effects between social and nonsocial scenes. One hypothesis is that, although explicit memory for social scenes is poorer, implicit memory, which has also been implicated in the orienting phase in addition to explicit memory (see discussion in Summerfield et al., 2006), may be better. Indeed, other work with a similar paradigm and healthy aging participants suggests that explicit contextual memory is not necessary for memory-guided attention to spatial locations in natural scenes; thus, memory-guided orienting can decorrelate from explicit memory measures (Salvato, Patai, McCloud, et al., 2016; Salvato, Patai, \& Nobre, 2016). Further research is necessary to explore this hypothesis. Another possibility is that the orienting phase was overall more difficult in trials with social scenes compared with nonsocial scenes. Previous literature reports larger validity effects with increased task difficulty for endogenous cues (arrow cues) compared with exogenous cues (Berger, Henik, \& Rafal, 2005). In light of this study, it is possible that, although memory for target locations in scenes with social distractors is "good enough" to detect targets at valid locations, participants pay a larger reorienting cost during invalid trials due to the difficulty of memory-guided orienting in the context of social distraction. Inclusion of neutral trials may help in exploring this hypothesis by determining if RTs are longer for neutral social compared with neutral nonsocial trials.

Crucially, investigating alpha desynchronization contralateral to learned target locations in response to centrally presented targets and scene cues during the orienting phase detected significant desynchronization in response to the presentation of central targets associated with nonsocial scenes, as well as the nonsocial scenes themselves. We had expected this finding, as alpha desynchronization of this kind has been reported in the context of memory-guided orienting to target locations in naturalistic scenes (Stokes et al., 2012). This was not the case for targets associated with social scenes or social scenes themselves, although this null lateralized alpha desynchronization needs to be caveated by the absence of an overall interaction effect of hemisphere and scene type. Importantly, the centrally presented targets were counterbalanced-although some participants saw a particular target and associated it with a social scene, other participants associated that same target with a nonsocial scene. It is unlikely, therefore, that differences arose due to the perceptual properties of the stimuli. These findings suggest that the effect of social distraction on explicit contextual memory extends to neural activity and specifically to lateralized alpha oscillations that have been shown to occur in response to perceptual cues (Haegens et al., 2011; van Ede et al., 2011; Romei et al., 2010; Wyart \& Tallon-Baudry, 2008; Thut et al., 2006; Worden et al., 2000), as well as memory cues (Stokes et al., 2012; Summerfield et al., 2011). Although the previous literature found alpha desynchronization in response to the scene cue with similar memory-guided orienting tasks using natural scenes (Stokes et al., 2012; Summerfield et al., 2011), in the current study, we extend this by showing for the first time even earlier similar lateralization, already following the presentation of an object cue that predicts the upcoming nonsocial scene cue. Crucially, EEG-detected lateralization that has been seen in response to lateralized targets (e.g., Patai et al., 2012) we have shown can be induced by central targets associated with lateralized locations, at least in the context of targets for which the memory trace had been laid down in nonsocial scenes.

Although we have interpreted these alpha modulations as an index of preparatory spatial attention for the upcoming target (in line with many other studies cited above), these modulations may also be associated with mnemonic retrieval operations alone (Hanslmayr et al., 2011). Although distinguishing between these alternatives remains an important target for future research, it is likely that both processes go hand in hand during memory-guided attentional orienting, provided that it is the mnemonic retrieval of the target location that steers the subsequent orienting of spatial attention. In addition, it is intriguing that, for scenes with social distractors, we do not detect alpha lateralization, yet do see strong behavioral memory-guided orienting effects. One explanation is there may have been parallel alpha lateralization to remembered social distractors and to targets that may have had a cancelling out effect. Although this could not be tested in the current study due to limited number of trials, this could be tested in the future. Another explanation is that net alpha lateralization is not required to carry out the behavioral effects of memory-guided attention orienting. Finally, there may be additional markers of net attention that correlate with the behavioral findings but were not investigated here.

Interestingly, moderation of the orienting validity effect by distractor type, as well as the finding of poorer explicit memory for social scenes, were still present when including longitudinal data following up participants approximately 1-3 weeks later (see supplementary online material https://osf.io/hm5cs/). These longitudinal results provide evidence against two possible hypotheses for the seemingly contrasting effects of distractor type 
between the memory phase and the orienting phase. One hypothesis is that, although explicit memory may initially be poorer for social scenes, in the approximately 30 intervening minutes until the orienting phase memory degrades more rapidly for nonsocial scenes, such that by the time participates engage in the task their memory has become poorer for nonsocial scenes. However, the longitudinal data provide evidence against this hypothesis, as poorer memory for social scenes appears to persist even 1-3 weeks later. Another hypothesis is that the distractor effect at the orienting phase is not due to distraction at encoding (during learning in the visual search phase), but rather distraction during retrieval (during the orienting phase) due to the presence of the social distractors within the scenes during the task. The longitudinal data again provide evidence against this hypothesis. In the longitudinal version of the orienting phase, the scenes and the distractors were not present when participants reacted to the target's appearance, which occurred on a gray screen (see supplementary online material https://osf.io/ $\mathrm{hm} 5 \mathrm{cs} /$ ). The only cue they were given was the centrally presented target, yet still there was a robust validity effect that appears to be moderated by distractor type.

In summary, the current study extends the literature on memory-guided attention orienting, and in particular the literature utilizing memories of spatial locations within natural scenes, by demonstrating the moderating effect of social distraction. Here, we show that an attention bias toward social stimuli, even when such stimuli are task irrelevant, results in poorer explicit contextual memory, which subsequently affects memory-guided attention orienting. Moreover, there is preliminary evidence to suggest these effects are long lasting, present even several weeks after memory encoding (see supplementary online material https://osf.io/hm5cs/). Finally, anticipatory lateralization of 8-12 Hz alpha oscillations following mnemonic cues is absent for targets associated with social scenes.

\section{Acknowledgments}

This research was funded by a James S. McDonnell Foundation Understanding Human Cognition Scholar Award (G. S.), the Rhodes Trust and the Clarendon Fund (B. R. D.), and a Wellcome Trust Senior Investigator Award 104571/Z/14/Z (A. C. N.) and supported by the NIHR Oxford Health Biomedical Research Centre. The Wellcome Centre for Integrative Neuroimaging was supported by core funding from the Wellcome Trust (203139/Z/16/Z).

Reprint requests should be sent to Gaia Scerif, Experimental Psychology Department, University of Oxford, Anna Watts Building, Oxford, OX2 6GG, United Kingdom, or via e-mail: gaia.scerif@psy.ox.ac.uk.

\section{REFERENCES}

Astle, D. E., \& Scerif, G. (2011). Interactions between attention and visual short-term memory (VSTM): What can be learnt from individual and developmental differences? Neuropsychologia, 49, 1435-1445.
Barr, D. J., Levy, R., Scheepers, C., \& Tily, H. J. (2013). Random effects structure for confirmatory hypothesis testing: Keep it maximal. Journal of Memory and Language, 68, 255-278.

Berger, A., Henik, A., \& Rafal, R. (2005). Competition between endogenous and exogenous orienting of visual attention. Journal of Experimental Psychology: General, 134, 207-221.

Burnham, K. P., \& Anderson, D. R. (2002). Model selection and multi-model inference: A practical information-theoretic approach. New York: Springer.

Chun, M. M., \& Jiang, Y. (1998). Contextual cueing: Implicit learning and memory of visual context guides spatial attention. Cognitive Psychology, 36, 28-71.

Chun, M. M., \& Turk-Browne, N. B. (2007). Interactions between attention and memory. Current Opinion in Neurobiology, 17, 177-184.

Doallo, S., Patai, E. Z., \& Nobre, A. C. (2013). Reward associations magnify memory-based biases on perception. Journal of Cognitive Neuroscience, 25, 245-257.

Doherty, B. R., Patai, E. Z., Duta, M., Nobre, A. C., \& Scerif, G. (2017). The functional consequences of social distraction: Attention and memory for complex scenes. Cognition, 158, 215-223.

Goldfarb, E. V., Chun, M. M., \& Phelps, E. A. (2016). Memoryguided attention: Independent contributions of the hippocampus and striatum. Neuron, 89, 317-324.

Griffin, I. C., \& Nobre, A. C. (2003). Orienting attention to locations in internal representations. Journal of Cognitive Neuroscience, 15, 1176-1194.

Haegens, S., Händel, B. F., \& Jensen, O. (2011). Top-down controlled alpha band activity in somatosensory areas determines behavioral performance in a discrimination task. Journal of Neuroscience, 31, 5197-5204.

Hanslmayr, S., Volberg, G., Wimber, M., Raabe, M., Greenlee, M. W., \& Bäuml, K.-H. T. (2011). The relationship between brain oscillations and BOLD signal during memory formation: A combined EEG-fMRI study. Journal of Neuroscience, 31, 15674-15680.

Harel, J., Koch, C., \& Perona, P. (2006). Graph-based visual saliency. Paper presented at the Neural Information Processing Systems (NIPS), Vancouver, British Columbia, Canada.

Hutchinson, J. B., \& Turk-Browne, N. B. (2012). Memoryguided attention: Control from multiple memory systems. Trends in Cognitive Sciences, 16, 576-579.

Jaeger, T. F. (2008). Categorical data analysis: Away from ANOVAs (transformation or not) and towards logit mixed models. Journal of Memory and Language, 59, 434-446.

Jensen, O., \& Mazaheri, A. (2010). Shaping functional architecture by oscillatory alpha activity: Gating by inhibition. Frontiers in Human Neuroscience, 4, 186.

Kelly, S. P., Lalor, E. C., Reilly, R. B., \& Foxe, J. J. (2006). Increases in alpha oscillatory power reflect an active retinotopic mechanism for distracter suppression during sustained visuospatial attention. Journal of Neurophysiology, 95, 3844-3851.

Kuhl, B. A., \& Chun, M. M. (2014). Memory and attention. In A. C. K. Nobre \& S. Kastner (Eds.), The Oxford handbook of attention (Vol. 1). Oxford: Oxford University Press.

Maris, E., \& Oostenveld, R. (2007). Nonparametric statistical testing of EEG- and MEG-data. Journal of Neuroscience Methods, 164, 177-190.

Nieuwenhuis, S., Forstmann, B. U., \& Wagenmakers, E.-J. (2011). Erroneous analyses of interactions in neuroscience: A problem of significance. Nature Neuroscience, 14, 1105-1107. 
Oostenveld, R., Fries, P., Maris, E., \& Schoffelen, J.-M. (2011). FieldTrip: Open source software for advanced analysis of MEG, EEG, and invasive electrophysiological data. Computational Intelligence and Neuroscience, 2011, 156869.

Patai, E. Z., Buckley, A., \& Nobre, A. C. (2013). Is attention based on spatial contextual memory preferentially guided by low spatial frequency signals? PLoS One, 8, e65601.

Patai, E. Z., Doallo, S., \& Nobre, A. C. (2012). Long-term memories bias sensitivity and target selection in complex scenes. Journal of Cognitive Neuroscience, 24, 2281-2291.

Percival, D. B., \& Walden, A. T. (1993). Spectral analysis for physical applications: Multitaper and conventional univariate techniques. Cambridge, MA: Cambridge University Press.

Romei, V., Gross, J., \& Thut, G. (2010). On the role of prestimulus alpha rhythms over occipito-parietal areas in visual input regulation: Correlation or causation? Journal of Neuroscience, 30, 8692-8697.

Rosen, M. L., Stern, C. E., Michalka, S. W., Devaney, K. J., \& Somers, D. C. (2016). Cognitive control network contributions to memory-guided visual attention. Cerebral Cortex, 26, 2059-2073.

Salvato, G., Patai, E. Z., McCloud, T., \& Nobre, A. C. (2016). Apolipoprotein 4 breaks the association between declarative long-term memory and memory-based orienting of spatial attention in middle-aged individuals. Cortex, 82, 206-216.

Salvato, G., Patai, E. Z., \& Nobre, A. C. (2016). Preserved memory-based orienting of attention with impaired explicit memory in healthy ageing. Cortex, 74, 67-78.

Stokes, M. G., Atherton, K., Patai, E. Z., \& Nobre, A. C. (2012). Long-term memory prepares neural activity for perception. Proceedings of the National Academy of Sciences, U.S.A., 109, E360-E367.
Summerfield, J. J., Lepsien, J., Gitelman, D. R., Mesulam, M. M., \& Nobre, A. C. (2006). Orienting attention based on longterm memory experience. Neuron, 49, 905-916.

Summerfield, J. J., Rao, A., Garside, N., \& Nobre, A. C. (2011). Biasing perception by spatial long-term memory. Journal of Neuroscience, 31, 14952-14960.

Thut, G., Nietzel, A., Brandt, S. A., \& Pascual-Leone, A. (2006). Alpha-band electroencephalographic activity over occipital cortex indexes visuospatial attention bias and predicts visual target detection. Journal of Neuroscience, 26, 9494-9502.

van Ede, F., de Lange, F., Jensen, O., \& Maris, E. (2011). Orienting attention to an upcoming tactile event involves a spatially and temporally specific modulation of sensorimotor alpha- and betaband oscillations. Journal of Neuroscience, 31, 2016-2024.

van Dijk, H., Schoffelen, J.-M., Oostenveld, R., \& Jensen, O. (2008). Prestimulus oscillatory activity in the alpha band predicts visual discrimination ability. Journal of Neuroscience, 28, 1816-1823.

van Ede, F., de Lange, F. P., \& Maris, E. (2012). Attentional cues affect accuracy and reaction time via different cognitive and neural processes. Journal of Neuroscience, 32, 10408-10412.

van Ede, F., Köster, M., \& Maris, E. (2012). Beyond establishing involvement: Quantifying the contribution of anticipatory $\alpha$ - and $\beta$-band suppression to perceptual improvement with attention. Journal of Neurophysiology, 108, 2352-2362.

Worden, M. S., Foxe, J. J., Wang, N., \& Simpson, G. V. (2000). Anticipatory biasing of visuospatial attention indexed by retinotopically specific-band electroencephalography increases over occipital cortex. Journal of Neuroscience, 20, RC63.

Wyart, V., \& Tallon-Baudry, C. (2008). Neural dissociation between visual awareness and spatial attention. Journal of Neuroscience, 28, 2667-2679. 